\title{
Comparative study of tributyltin toxicity on two bacteria of the genus Bacillus
}

\author{
J.D. Martins ${ }^{\text {a }}$, A.S. Jurado ${ }^{\text {a,b,*, }}$ A.J.M. Moreno ${ }^{\text {c }}$, V.M.C. Madeira ${ }^{\text {a }}$ \\ a Centro de Neurociências, Departamento de Zoologia, Universidade de Coimbra, 3000 Coimbra, Portugal \\ b Departamento de Bioquímica, Universidade de Coimbra, 3000 Coimbra, Portugal \\ ${ }^{\mathrm{c}}$ Instituto do Mar, Universidade de Coimbra, 3000 Coimbra, Portugal
}

Received 15 April 2005; accepted 17 June 2005

Available online 2 August 2005

\begin{abstract}
Tributyltin is a potent biocide mainly used in marine antifouling paints. Owing to its widespread distribution in coast areas and its high toxicity to aquatic organisms, the use of this compound is generally restricted and under government regulation. Despite of that, it persists in the aquatic environment. Organotins used in industry have also been detected in terrestrial environments. The persistence and high lipophilicity explain bioaccumulation. The role of bacteria in recycling organic matter prompted us to study the interaction of tributyltin with two ubiquitous bacilli, B. stearothermophilus and B. subtilis, proposed as biological indicators of pollutants with ecological impact. These bacteria have been used as suitable models for the study of toxicity mechanisms of unselective lipophilic compounds (e.g., DDT and endosulfan). Drug effects on growth parameters, oxygen consumption and membrane organization were assessed. Bacteria growth in a liquid complex medium was disturbed by concentrations of TBT as low as $25 \mathrm{nM}$ $\left(8 \mu \mathrm{g} \mathrm{L}^{-1}\right)$, close to the concentration in polluted environments. The respiratory activity is affected by TBT in both microorganisms. Membrane organization, assessed by fluorescence polarization of two fluidity probes, 1,6-diphenyl-1,3,5-hexatriene (DPH) and a propionic acid derivative (DPH-PA), was also perturbed by the xenobiotic. Alterations on growth, oxygen consumption and physical properties of membrane lipids are stronger in B. stearothermophilus as compared to B. subtilis. A putative relationship between growth inhibition and respiratory activity impairment induced by TBT and its effects on the physical behaviour of bacterial membrane lipids is suggested.
\end{abstract}

(C) 2005 Elsevier Ltd. All rights reserved.

Keywords: Organotin compounds; Fluorescence polarization; Bacterial growth; Lipid membrane; Bacillus stearothermophilus; Bacillus subtilis

\section{Introduction}

Toxicological concerns of TBT emerged from its use as a biocide in antifouling paints, leading to its ubiqui-

Abbreviations: TBT, tributyltin; NADH, nicotinamide adenine dinucleotide, reduced form; TMPD, $N, N, N^{\prime}, N^{\prime}$-tetramethyl- $p$ phenylenediamine.

Corresponding author. Address: Centro de Neurociências, Departamento de Zoologia, Universidade de Coimbra, 3000 Coimbra, Portugal. Tel.: +351 239 834729; fax: +351 239826798 .

E-mail address: asjurado@ci.uc.pt (A.S. Jurado). tous distribution on aquatic environments (White et al., 1999; Rüdel, 2003). Furthermore, industrial utilization in preservation of wood, cotton textiles, paper, leather and paints (White et al., 1999) contributes for the presence of organotin compounds in terrestrial environments (White et al., 1999; Huang and Matzner, 2004).

Reports of TBT toxicity to organisms belonging to the five taxonomic kingdoms have been reported (White et al., 1999; White and Tobin, 2004; Smith et al., 2003; Jensen et al., 2004), endocrine disruption, (Schulte-Oehlmann et al., 1997) and mitochondria function impairment (Jurkiewicz et al., 2004) being the main toxic 
effects. However, the molecular mechanisms underlying the toxicity of TBT are far from full understanding. Due to the high lipophilicity of TBT $\left(\log K_{\text {ow }}\right.$ between 2.3 and 4.4, depending on physico-chemical conditions; (Rüdel, 2003), biological membranes have been considered putative targets for its action (White et al., 1999; Gadd, 2000).

Microorganisms have been used as powerful tools to assess in vitro the toxicity of several environmental pollutants, namely polycyclic aromatic hydrocarbons, industrial solvents and agrochemicals (Sikkema et al., 1995). On the other hand, data of the toxic effects on microorganisms (the basic levels of organization of soil and aquatic communities) may be used to define upper limits for concentration of pollutants and to predict environmental toxicity risks. B. stearothermophilus has been used as a suitable model for the toxicity assessment of drugs (Luxo et al., 2000; Rosa et al., 2000; Monteiro et al., 2003) and pesticides (Donato et al., 1997; Martins et al., 2003). Growth, cell viability and oxygen consumption have proved to be sensitive biological parameters to assess chemical toxicity of lipophilic xenobiotics, often associated with disturbance of the membrane lipid organization. This study aims to collect data of similar studies with TBT to establish and further develop $B$. stearothermophilus as a bacterial model for screening tests of chemical toxicity, and also to compare the relative sensibility to xenobiotics of two philogenetically related organisms with distinct living conditions $(B$. subtilis, a mesophile and B. stearothermophilus, a thermophile). The choice of these bacteria was also motivated because data of TBT action on organisms of terrestrial environment are scarce. The effects of TBT on growth, oxygen consumption and in the physical behaviour of bacterial lipid membranes of the two species of Bacillus will be studied.

\section{Materials and methods}

\subsection{Chemicals}

Tributyltin chloride (TBT), 1,6-diphenyl-1,3,5-hexatriene (DPH) and 1,6-diphenyl-1,3,5-hexatriene propionic acid (DPH-PA) were obtained from Sigma Chemical co.

\subsection{Cultures}

The strains of B. stearothermophilus and B. subtilis (ATCC 6051) and the conditions for their maintenance and growth have been described previously (Jurado et al., 1987). TBT from concentrated ethanolic solutions, was added to the growth medium (diluted LBroth) in order to obtain concentrations from 25 to $500 \mathrm{nM}$. Growth was measured by turbidimetry at
$610 \mathrm{~nm}$ in a Bausch \& Lomb Spectronic 21 spectrophotometer.

\subsection{Oxygen consumption}

Obtainment of protoplasts, protein quantification, and monitoring of oxygen consumption were described elsewhere (Donato et al., 1997). Shortly, protoplasts were obtained by treatment of cells collected at the middle of exponential growth phase. Protein was quantified by the biuret method using serum bovine albumin for calibration. A Clark oxygen electrode was used to measure the oxygen consumption at 37 and $40{ }^{\circ} \mathrm{C}$ for B. subtilis and $B$. stearothermophilus, respectively. TBT was added to the protoplasts ( $0.5 \mathrm{mg}$ protein) $4 \mathrm{~min}$ before the substrate (either NADH $10 \mathrm{mM}$ or ascorbate $10 \mathrm{mM} / \mathrm{TMPD} 600 \mu \mathrm{M}$ ). Oxygen consumption is expressed as $\%$ of the control, corresponding to $0 \mathrm{nM}$ TBT (the solvent DMSO had no effects at the used volumes).

\subsection{Lipid analysis}

Cells grown up to the beginning of the stationary phase were harvested by low-speed centrifugation and washed three times with buffer $(10 \mathrm{mM}$ Tris- $\mathrm{Cl}, \mathrm{pH}$ 7.0). The lipids were extracted by the Bligh and Dyer method (1959) and quantified by measuring the amount of inorganic phosphate (Bartlett, 1959) after hydrolysis of the extracts at $180{ }^{\circ} \mathrm{C}$ in $70 \% \mathrm{HClO}_{4}$ (Böttcher et al., 1961). The polar lipids were isolated by preparative thin layer chromatography, as previously described (Jurado et al., 1991).

\subsection{Liposomes}

Aliquots from lipid solutions in $\mathrm{CHCl}_{3}$ (polar lipid extract) containing $1.34 \mathrm{mg}$ of lipid were evaporated to dryness on a rotary evaporator. The dry residues were hydrated under $\mathrm{N}_{2}$ atmosphere at $55^{\circ} \mathrm{C}$ by gentle shaking with $5 \mathrm{ml}$ of $50 \mathrm{mM} \mathrm{KCl}$ and $10 \mathrm{mM}$ Tris-maleate (pH 7.0) and multilamellar vesicles were obtained. Then, the suspensions were vortexed for $1 \mathrm{~min}$ to disperse aggregates.

\subsection{Fluorimetric measurements}

The fluidity probes DPH and DPH-PA in dimethylformamide were injected (few $\mu$ l) into liposomes suspensions ( $345 \mu \mathrm{M}$ in phospholipid), as previously described (Antunes-Madeira et al., 1994) to give a lipid/probe molar ratio of 400 . The mixture was vigorously vortexed for $10 \mathrm{~s}$, and then TBT was added from concentrated ethanolic solutions. The mixture was incubated at 55 or $37^{\circ} \mathrm{C}$ (respectively for $B$. stearothermophilus or $B$. subtilis) in the dark, for a period of $18-20 \mathrm{~h}$ to reach 
equilibrium. Control samples received equivalent volumes of dim-ethylformamide and ethanol. Added solvent volumes (few $\mu$ l) had negligible effects on measurements.

The fluorimetric measurements were performed with a Perkin-Elmer spectrofluorimeter, model MPF-66, with a thermostated cell holder. The excitation was set at $336 \mathrm{~nm}$ and the emission at $450 \mathrm{~nm}$ ( $5 \mathrm{~nm}$ excitation and $6 \mathrm{~nm}$ band pass).

All fluorescence measurements were corrected for the contribution of light scattering by using appropriate blanks without added probes. The degree of fluorescence polarization $(P)$ was calculated according to Shinitzky and Barenholz (1978) from the equation:

$P=\frac{I_{\|}-G I_{\perp}}{I_{\|}+G I_{\perp}}$

where $I_{\|}$and $I_{\perp}$ are the intensities of the light emitted with its polarization plane parallel $(\|)$ and perpendicular $(\perp)$ to that of exciting beam. $G$ is the correction factor for instrument polarization, given by the ratio of the vertically to the horizontally polarized emission components when the excitation light is polarized in the horizontal plane.

\section{Results}

\subsection{Effect of TBT on the growth of B. stearothermophilus and B. subtilis}

B. stearothermophilus and B. subtilis were grown at 65 and $37^{\circ} \mathrm{C}$, respectively (i.e. in optimal temperature ranges) in a complex medium (diluted L-Broth) with an endogenous concentration of $0.115 \mu \mathrm{M} \mathrm{Ca}^{2+}$ (Jurado et al., 1987). To this basal medium, TBT was added from a concentrated ethanolic solution to obtain concentrations ranging from 25 to $500 \mathrm{nM}$; control cultures were grown in a medium without TBT but with the sol- vent (ethanol). The solvent by itself has no effect on growth. Concentration-dependent alterations of growth were induced by TBT in the two bacilli (Table 1). Increasing concentrations of TBT added to the growth medium (in the range of $25-500 \mathrm{nM}$ ) increasingly inhibited growth (Fig. 1 and Table 1). However, in the range of $25-150 \mathrm{nM}$ TBT, the growth parameters were differently affected in the two species of Bacillus. In B. stearothermophilus, the main parameter affected by TBT was the maximal cell density reached in the stationary phase, which showed a progressive decrease with increasing concentrations of the compound. The specific growth rate was not significantly altered in the range of 25 $100 \mathrm{nM}$ TBT, but showed a drastic decrease with the addition of concentrations above $150 \mathrm{nM}$ TBT. In contrast, the maximal cell density of cultures of B. subtilis showed a slight decrease $(7 \%)$ by the addition of TBT (in the range of 25-150 nM), whereas the specific growth rate progressively decreased as TBT concentration increased in the same range. At concentrations above $150 \mathrm{nM}$ TBT, a significant decrease of the bacterial yield was also noticed. In both species, effects of TBT on the lag time were not detected.

\subsection{Effect of TBT on the oxygen consumption of B. stearothermophilus and B. subtilis protoplasts}

Protoplasts prepared from cells of B. stearothermophilus and $B$. subtilis grown in the basal medium, in the optimal temperature ranges $\left(65\right.$ and $37^{\circ} \mathrm{C}$, respectively), were used to assess the effect of TBT on the oxygen consumption rate. The respiratory activity was measured at $40^{\circ} \mathrm{C}$ for B. stearothermophilus and $37^{\circ} \mathrm{C}$ for $B$. subtilis, and the protoplasts were incubated during 4 min with the desired TBT concentration prior to the addition of the respiratory substrate. The oxygen consumption promoted by the addition of NADH to protoplasts of both bacteria decreased with increasing TBT concentrations (Fig. 2). This effect is

Table 1

Specific growth rate, and maximum cell density of cultures of B. stearothermophilus and B. subtilis grown in media with different concentrations of TBT

\begin{tabular}{|c|c|c|c|c|}
\hline \multirow[t]{2}{*}{ TBT (nM) } & \multicolumn{2}{|c|}{ Specific growth rate $\left(\mathrm{h}^{-1}\right)^{\mathrm{a}}$} & \multicolumn{2}{|c|}{ Maximum cell density ( $\%$ of control $)^{\mathrm{a}}$} \\
\hline & Stearothermophilus & Subtilis & Stearothermophilus & Subtilis \\
\hline 0 & $2.361 \pm 0.129(7)$ & $1.209 \pm 0.114(5)$ & 100 & 100 \\
\hline 25 & $2.447 \pm 0.163(4)^{\text {n.s. }}$ & $0.975 \pm 0.080(3)^{*}$ & $94.8 \pm 1.2(4)^{*}$ & $94.1 \pm 3.3(3)^{\text {n.s. }}$ \\
\hline 50 & $2.295 \pm 0.096(4)^{\text {n.s.,n.s. }}$ & $0.960 \pm 0.030(3)^{* *}$, n.s. & $81.0 \pm 4.0(4)^{* * *}$, ooo & $91.3 \pm 3.0(4)^{\text {n.s.,n.s. }}$ \\
\hline 100 & $2.103 \pm 0.080(4)^{*}$, n.s. & $0.826 \pm 0.008(3)^{* * *, \text { n.s. }}$ & $63.5 \pm 2.9(5)^{* * *}$, ooo & $90.9 \pm 5.9(7)^{\text {n.s.,n.s. }}$ \\
\hline 150 & $1.339 \pm 0.120(5)^{* * *, \text { ooo }}$ & $0.725 \pm 0.056(3)^{* * *, n . s .}$ & $54.1 \pm 2.9(6)^{* * *, \text { ooo }}$ & $93.4 \pm 2.8(3)^{\text {n.s..,n.s. }}$ \\
\hline 250 & $0.808 \pm 0.022(3)^{* * *, \text { ooo }}$ & $0.670 \pm 0.113(3)^{* * *, \text { n.s. }}$ & $20.7 \pm 2.1(5)^{* * *, \text { ooo }}$ & $66.6 \pm 13.2(3)^{* * *, o o}$ \\
\hline 500 & n.d. & $0.508 \pm 0.065(3)^{* * *}$, n.s. & n.d. & $45.0 \pm 11.3(3)^{* * *, o}$ \\
\hline
\end{tabular}

${ }^{a}$ Results are means \pm standard deviation of at least three independent experiments and comparisons were performed using Tukey's multiple comparison test, for the following paired observations: cultures grown with different concentrations of TBT vs control cultures (n.s. not significant, $\left.{ }^{*} p<0.05{ }^{* *} p<0.01 ;{ }^{* * *} p<0.001\right)$ and cultures grown with $50,100,150,250$ and $500 \mathrm{nM}$ TBT vs cultures grown with $25,50,100,150$ and $250 \mathrm{nM}$ TBT, respectively (n.s. not significant, ${ }^{\circ} p<0.05 ;{ }^{\circ o} p<0.01,{ }^{\circ o o} p<0.001$ ). 


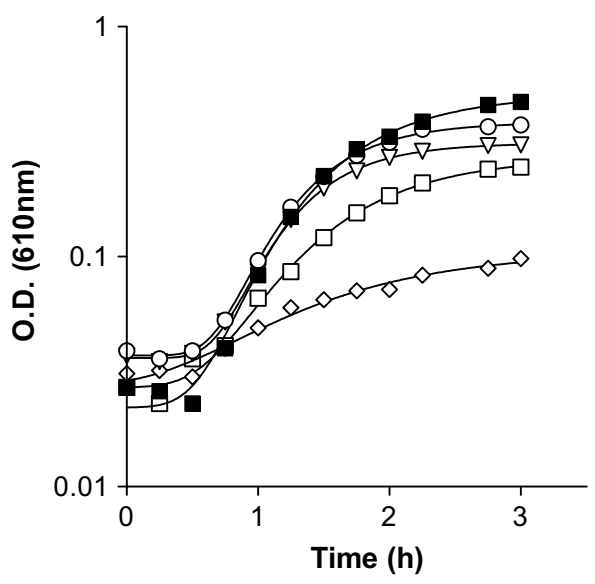

Fig. 1. TBT effects on the growth of B. stearothermophilus at $65^{\circ} \mathrm{C}$. Cells were grown in a basal medium (dilute L-Broth) without TBT (ם) and with $50 \mathrm{nM}(\bigcirc), 100 \mathrm{nM}(\nabla), 150 \mathrm{nM}(\square)$ and $250 \mathrm{nM}(\diamond)$ TBT. The results are typical of at least three independent experiments.

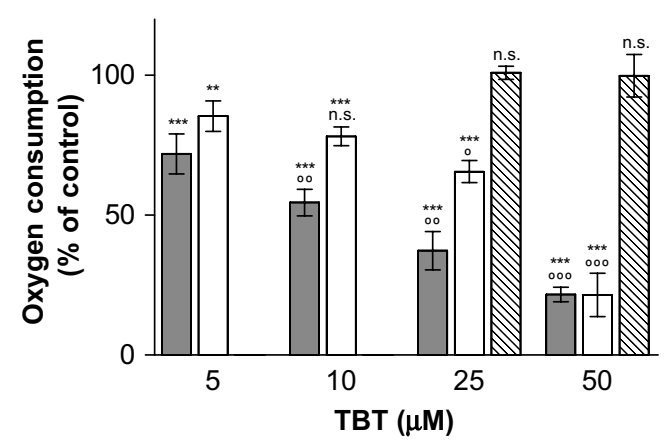

Fig. 2. TBT effects on the oxygen consumption rate of B. stearothermophilus (grey and listed bars) and B. subtilis (white bars) protoplasts after 4 min of incubation at 40 and $37^{\circ} \mathrm{C}$, respectively, expressed as $\%$ of the control (protoplasts incubated for $4 \mathrm{~min}$ without xenobiotic, in the presence of a few microliters of dimethylsulfoxide, the TBT solvent); $10 \mathrm{mM}$ NADH (grey and white bars) or $10 \mathrm{mM}$ ascorbate$600 \mu \mathrm{M}$ TMPD (listed bars) was used as respiratory substrate. Results are means \pm standard deviation of at least three independent experiments and comparisons were performed using Tukey's multiple comparison test, for the following paired observations: protoplasts with $5,10,25$ and $50 \mu \mathrm{M}$ TBT vs control protoplasts (n.s., not significant; ${ }^{* *} p<0.01 ;{ }^{* * *} p<0.001$ ); protoplasts with 10,25 and $50 \mu \mathrm{M}$ TBT vs protoplasts with 5,10 and $25 \mu \mathrm{M}$ TBT, respectively (n.s., not significant; ${ }^{\circ} p<0.05$; ${ }^{\circ o} p<0.01$; ${ }^{\circ o o} p<0.001$ ); protoplasts of $B$. stearothermophilus with $5,10,25$ and $50 \mu \mathrm{M}$ TBT vs protoplasts of $B$. subtilis with the same TBT concentrations (results are statistically different with $p<0.001$ for 10 and $25 \mu \mathrm{M}$ TBT and n.s. for 5 and $50 \mu \mathrm{M}$ TBT; in sake of clarity, symbols were not shown in figure).

significantly higher $(p<0.001)$ in protoplasts of $B$. stearothermophilus as compared with $B$. subtilis, except for the extreme TBT concentrations. TBT did not have any effect when ascorbate/TMPD supported the respiratory rate of B. stearothermophilus protoplasts (Fig. 2). This substrate is not suitable for $B$. subtilis protoplasts owing to a different terminal oxidase, probably a quinol oxidase instead of a standard cytochrome oxidase (Lauraeus and Wikström, 1993). As expected, the oxygen consumption supported by NADH or ascorbate-TMPD was completely impaired by the addition of $\mathrm{KCN}$ $(1 \mathrm{mM})$, as consequence of complete inhibition of terminal oxidase.

\subsection{Physical effects of TBT on bilayers of B. stearothermophilus and B. subtilis polar lipids}

To investigate if TBT toxic action on bacteria could result from perturbations of membrane lipid organization, fluorescence polarization measurements were performed using DPH and DPH-PA as fluidity probes, incorporated in liposomes prepared with the polar lipids of B. stearothermophilus and B. subtilis (grown at 65 and $37^{\circ} \mathrm{C}$, respectively). The rotational motions of the probes that result in depolarization of fluorescence are tightly coupled to acyl chain orientational fluctuations (Lentz et al., 1976) and, consequently, reflect the degree of molecular packing of the lipids in the probe bilayer environment. Thus the term fluidity, opposite to structural membrane order, will be used here in an operational sense and defined as being directly proportional to the reciprocal of fluorescence polarization. Monitoring of TBT induced disturbance in lipid packing across the bilayer thickness was achieved by the use of DPH, buried in the hydrophobic core of the bilayer (Shinitzky and Barenholz, 1978), and DPH-PA, anchored close to the lipid-water interphase because of its charged propionic group (Trotter and Storch, 1989). As shown in Fig. 3, temperature-dependent DPH and DPH-PA fluorescence polarization exhibits a sharp decrease over a range of about $20^{\circ} \mathrm{C}$. This indicates a broad transition from the gel to liquid-crystalline phase undergone by the bilayers prepared with the mixtures of bacterial polar lipids. Identical results were previously obtained with dispersions of the polar lipids of B. stearothermophilus by DSC and spectrofluorimetry, using different fluorescent probes, and have been explained by the heterogeneous composition of the lipid preparations (Jurado et al., 1991). Comparing the polarization values detected in the same preparation by the two probes at the same temperature, it is evident an increase of order from the core of the bilayer, monitored by DPH, to the outer regions, where DPH-PA distributes, in agreement with classical reports (Tilley et al., 1979).

At temperatures below the phase transition, i.e. up to $20{ }^{\circ} \mathrm{C}$ for B. stearothermophilus lipid preparations and up to $10^{\circ} \mathrm{C}$ for $B$. subtilis, the fluorescence polarization of DPH and DPH-PA decreases with increasing concentrations of TBT (in the range of 1:12 to 1:4 TBT:lipid molar ratio), as shown in Fig. 3. This reflects an increase of fluidity induced by TBT across the bilayer thickness. However, the decrease of order induced by the same TBT concentration (e.g., 1:6 TBT:lipid molar ratio) is much more significant in the outer regions of the bilayer, monitored by DPH-PA, than in the hydrophobic core, monitored by DPH. The thermograms of membrane 

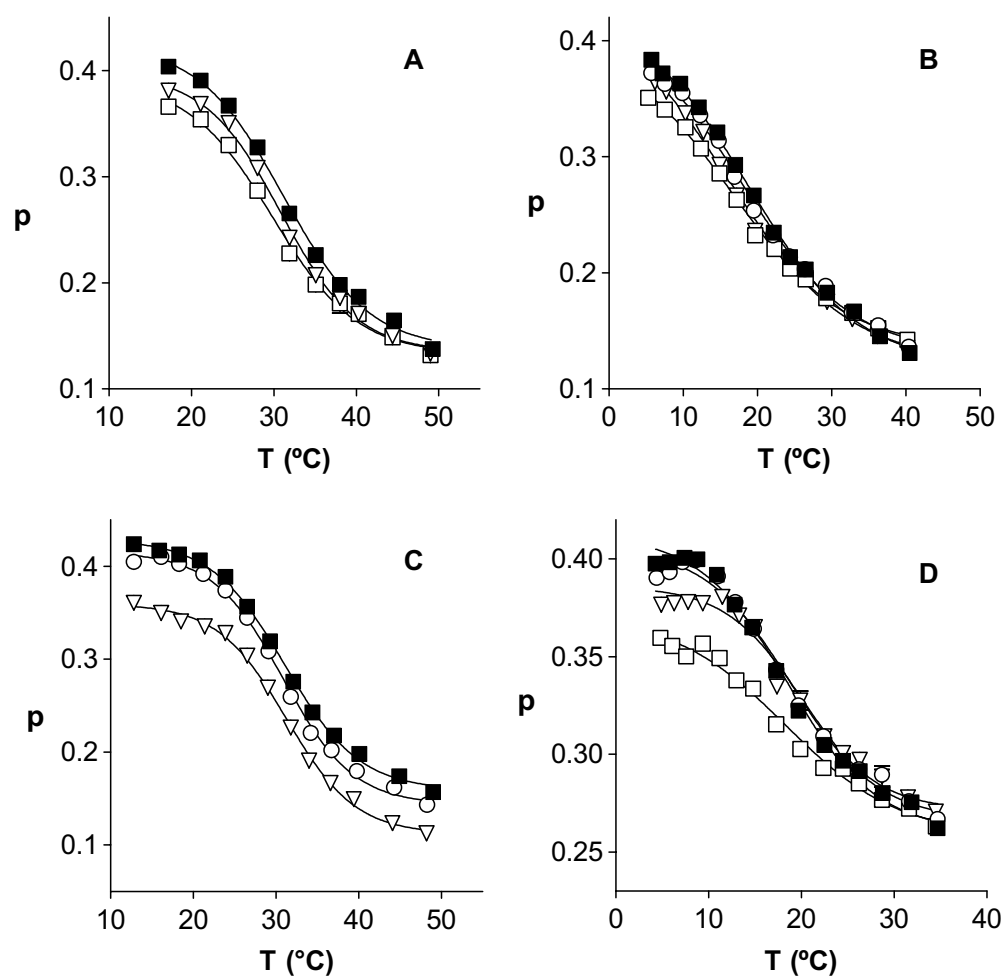

Fig. 3. Thermograms of fluorescence polarization (P) of DPH (A and B) and DPH-PA (C and D) in liposomes prepared with the polar lipids of cells of B. stearothermophilus (A and C) and B. subtilis (B and D) grown in the basal medium, at 65 and $37^{\circ} \mathrm{C}$, respectively. Liposomes were incubated without $(\square)$ or with 1:12 $(\bigcirc)$, 1:6 $(\nabla)$ and 1:4 $(\square)$ TBT:lipid molar ratio. The thermotropic profiles are typical assays of at least three independent experiments. Polarization values are means of three readings of fluorescence intensities, for the same assay, and error bars (SD) are not represented since, for most points, they are encompassed by the size of the symbols.

preparations of both bacteria show that the addition of TBT also induced a concentration-dependent decrease of fluorescence polarization along the phase transition temperature range, that is when ordered and disordered lipid domains coexist. This effect was also more apparent in the outer membrane regions, as reported by DPH-PA. In the fluid phase (above $40^{\circ} \mathrm{C}$ for B. stearothermophilus lipid dispersions and above $28^{\circ} \mathrm{C}$ for $B$. subtilis), TBT differently affected the lipid membranes of the two bacilli species. It induced a disordering effect in the outer regions of $B$. stearothermophilus lipid bilayers but had no effect on $B$. subtilis membrane lipids. Additionally, when a concentration-dependent effect was detected in both bacteria, the lipids from B. stearothermophilus showed consistently an increased susceptibility to TBT, i.e. a higher perturbation for the same TBT concentration. This effect is highly reproducible and its actual significance can be better assessed comparing the increase of disorder induced isothermally by TBT to that resulting from a decrease of temperature. For example, at $13{ }^{\circ} \mathrm{C}, P$ detected by DPH-PA in $B$. stearothermophilus lipid bilayers (Fig. 3C) decreases from 0.424 to 0.357 with the addition of TBT 1:6 (drug:lipid molar ratio). An identical decrease of $P$ is induced in control liposomes by a decrease of $13.3^{\circ} \mathrm{C}$. As was shown, the physical impact of TBT interaction with membranes clearly depends on the lipid phase and on the lipid composition.

\section{Discussion}

The wide range of organisms, bacteria included, affected by the toxic effects of TBT (White et al., 1999; Alzieu, 2000; Petersen and Gustavson, 2000; Qun-Fang et al., 2002; Smith et al., 2003; Jensen et al., 2004; White and Tobin, 2004) suggests that molecular cell components common to all living systems, namely biomembranes, may constitute the main target of this lipophilic xenobiotic. Prokaryotic cells are particularly useful to study the molecular toxicity of membraneactive compounds, offering advantage over eukaryotic cells owing to the simple membrane organization. Our experience with the use of a strain of B. stearothermophilus as a model to evaluate the toxicity of lipophilic drugs (Luxo et al., 2000; Rosa et al., 2000; Monteiro et al., 2003) and pollutants (Donato et al., 1997; Martins et al., 2003) prompted us to use this bacterium to further elucidate the membrane mediated toxic effects of TBT. Growth of B. stearothermophilus is very sensitive to xenobiotics inducing membrane perturbations and growth inhibition reasonably correlates with other 
bioindicators of chemical stress in eukaryotic cells, e.g., impairment of respiratory activity of rat liver mitochondria (Donato et al., 1997). A mesophilic species ( $B$. subtilis) has been also used here as a model for toxicity assessment, to obtain additional information by combined experimental data.

A growth inhibition, as a function of TBT concentration added to the culture medium, occurs with both bacteria, although with different sensitivities. The amount of growth of cultures of $B$. stearothermophilus was more depressed by the addition of TBT, as compared with $B$. subtilis. Thus, the maximum cell density decreased to $20.7 \%$ of the control, in cultures of B. stearothermophilus for $250 \mathrm{nM}$ TBT and to $66.6 \%$, in cultures of B. subtilis for the same concentration of TBT. A concentrationdependent inhibitory effect on the oxygen consumption supported by NADH in protoplasts of both bacteria was also detected. This effect was significantly greater, in the range of 5-25 $\mu \mathrm{M}$, in protoplasts of B. stearothermophilus, as compared with $B$. subtilis. Since the oxygen consumption elicited by ascorbate-TMPD revealed insensitive to the addition of TBT in B. stearothermophilus protoplasts, we concluded that TBT interacts with the respiratory system of this bacterium at level(s) preceding the terminal oxidase segment. The same conclusion is open for B. subtilis, since ascorbate-TMPD is not suitable as substrate for the terminal oxidase, putatively a quinol oxidase, at variance with a cytochrome $\mathrm{c}$ oxidase in B. stearothermophilus (Lauraeus and Wikström, 1993). This is related with the absence of the cytochrome c bridge of the respiratory system in $B$. subtilis grown in a rich glucose medium (Lauraeus and Wikström, 1993). Although further studies to individually assess the enzymatic activities of the respiratory complexes are needed to elucidate TBT effects on the bioenergetics of these two species of Bacillus, data suggest that perturbations of the respiratory activity may underlie the impairment of bacterial growth, as suggested for other microorganisms (Gadd, 2000) and in agreement with results obtained with mitochondria (Stockdale et al., 1970; Gogvadze et al., 2002).

Lipid bilayer structure and dynamics play a pivotal role for membrane proper functioning, as a selective barrier and a matrix for enzymes (Bloom et al., 1991). Thus, the cytotoxic effects of a variety of drugs and pollutants are suggested to result from their incorporation into the lipid bilayer and a consequence of the ability to affect and modulate lipid membrane physical properties (Sikkema et al., 1995). In this toxicological context, physical studies with dispersions of bacterial polar lipids were performed to elucidate TBT effects on the membrane lipid organization, putatively related with TBT induced impairment of growth and respiratory activity of the bacteria. The different physical effects promoted by TBT on membranes prepared with the polar lipids of $B$. stearothermophilus and B. subtilis may reflect differ- ences in the membrane lipid composition of the two species of Bacillus. (Bishop et al., 1977; Minnikin and Goodfellow, 1981; Martins et al., 1990; Jurado et al., 1991; Klein et al., 1999) and are in accordance with studies with model membranes prepared with different synthetic lipids (Ambrosini et al., 1991; Chicano et al., 2001). In spite of the differences in the thermotropic profiles, most relevant is the general disordering effect induced by TBT on both bacterial lipid preparations, mainly affecting the interfacial region of the bilayer monitored by DPH-PA, in accordance with previous studies performed with phosphatidylcholine liposomes (Ambrosini et al., 1991; Chicano et al., 2001) suggesting that organotin compounds are located in the upper part of the phospholipid bilayer (Chicano et al., 2001).

At present, it is not possible to provide a full explanation for the different effects of TBT on B. stearothermophilus and B. subtilis, but consistent perturbations on growth, respiration and membrane physical properties are detected in both species, although the effects are generally stronger in B. stearothermophilus. This supports the idea that $B$. stearothermophilus is a good tool to model toxicity studies with the advantage of a rapid growth performed at temperatures that avoid contamination with other species. Since TBT effects on membranes depend on the lipid composition, it may be useful to identify strategies that can improve bacterial resistance to the toxic effects of TBT in the perspective of using microorganisms in bioremediation.

\section{References}

Alzieu, C., 2000. Impact of tributyltin on marine invertebrates. Ecotoxicology 9, 71-76.

Ambrosini, A., Bertoli, E., Tanfani, F., Zolese, G., 1991. Effect of the fungicides tributyltin acetate and tributyltin chloride on multilamellar liposomes: fluorescence studies. Chemistry and Physics of Lipids 59, 189-197.

Antunes-Madeira, M.C., Videira, R.A., Madeira, V.M.C., 1994. Effects of parathion on membrane organization and its implications for the mechanisms of toxicity. Biochimica et Biophysica Acta 1190, 149-154.

Bartlett, G.R., 1959. Phosphorus assay in column chromatography. Journal of Biological Chemistry 234, 466-468.

Bishop, D.G., Op Den Kamp, J.A.F., Van Deenen, L.L.M., 1977. The distribution of lipids in the protoplast membranes of Bacillus subtilis. European Journal of Biochemistry 80, 381-391.

Bligh, E.G., Dyer, W.J., 1959. A rapid method of total lipid extraction and purification. Canadian Journal of Biochemistry and Physiology $37,911-917$.

Bloom, M., Evans, E., Mouritsen, O.G., 1991. Physical properties of the fluid lipid-bilayer component of cell membranes: a perspective. Quarterly Reviews of Biophysics 24, 293-397.

Böttcher, C.J.F., van Gent, C.M., Pries, C., 1961. A rapid and sensitive submicro phosphorus determination. Analytica Chimica Acta 24, 203-204.

Chicano, J.J., Ortiz, A., Teruel, J.A., Aranda, F.J., 2001. Organotin compounds alter the physical organization of phosphatidylcholine membranes. Biochemica et Biophysica Acta 1510, 330-341. 
Donato, M.M., Jurado, A.S., Antunes-Madeira, M.C., Madeira, V.M.C., 1997. Bacillus stearothermophilus as a model to evaluate membrane toxicity of a lipophilic environmental pollutant (DDT). Archives of Environmental Contamination and Toxicology 33, 109-116.

Gadd, G.M., 2000. Microbial interactions with tributyltin compounds: detoxification, accumulation and environmental fate. The Science of the Total Environment 258, 119-127.

Gogvadze, V., Stridh, H., Orrenius, S., Cotgreave, I., 2002. Tributyl causes cytochrome $c$ release from isolated mitochondria by two discrete mechanisms. Biochemical and Biophysical Research Communications 292, 904-908.

Huang, J.H., Matzner, E., 2004. Degradation of organotin compounds in organic and mineral forest soils. Journal of Plant Nutrition and Soil Science 167, 33-38.

Jensen, H.F., Holmer, M., Dahllöf, I., 2004. Effects of tributyltin on the seagrass Ruppia maritime. Marine Pollution Bulletin. Available from: <www.sciencedirect.com $>$.

Jurado, A.S., Santana, A.C., Costa, M.S., Madeira, V.M.C., 1987. Influence of divalent cations on the growth and morphology of Bacillus stearothermophilus. Journal of General Microbiology 133, 507-513.

Jurado, A.S., Pinheiro, T.J.T., Madeira, V.M.C., 1991. Physical studies on membrane-lipids of Bacillus stearothermophilus. Temperature and calcium effects. Archives of Biochemistry and Biophysics 289, 167-179.

Jurkiewicz, M., Averill-Bates, D.A., Marion, M., Denizeau, F., 2004. Involvement of mitochondrial and death receptor pathways in tributyltin-induced apoptosis in rat hepatocytes. Biochimica et Biophysica Acta 1693, 15-27.

Klein, W., Weber, M.H.W., Marahiel, M.A., 1999. Cold shock response of Bacillus subtilis: isoleucine-dependent switch in the fatty acid branching pattern for membrane adaptation to low temperatures. Journal of Bacteriology 181 (17), 5341-5349.

Lauraeus, M., Wikström, M., 1993. The terminal quinol oxidases of Bacillus subtilis have different energy conservation properties. The Journal of Biological chemistry 268, 11470-11473.

Lentz, B.R., Barenholz, Y., Thompson, T.E., 1976. Fluorescence depolarisation studies of phase transitions and fluidity in phospholipid bilayers. 1. Single component phosphatidylcholine liposomes. Biochemistry 15, 4521-4528.

Luxo, C., Jurado, A.S., Madeira, V.M.C., 2000. Toxicity assessment of tamoxifen by means of a bacterial model. Applied Biochemistry and Biotechnology 87, 219-232.

Martins, L.O., Jurado, A.S., Madeira, V.M.C., 1990. Biochimica et Biophysica Acta 1045, 17-20.

Martins, J.D., Monteiro, J.P., Antunes-Madeira, M.C., Jurado, A.S., Madeira, V.M.C., 2003. Use of B. stearothermophilus as a model to evaluate toxicity of the lipophilic environmental pollutant endosulfan. Toxicology in Vitro 17, 595-601.
Minnikin, D.E., Goodfellow, M., 1981. The Aerobic EndosporeForming Bacteria: Special Publications of the Society for General Microbiology. Academic Press, London, New York, Toronto, Sidney, San Francisco (Chapter 4).

Monteiro, J.P., Martins, J.D., Luxo, P.C., Jurado, A.S., Madeira, V.M.C., 2003. Molecular mechanisms of the metabolite 4-hydroxytamoxifen of the anticancer drug tamoxifen: use of a model microorganism. Toxicology in Vitro 17, 629-634.

Petersen, S., Gustavson, K., 2000. Direct toxic effects of TBT on natural enclosed phytoplankton at ambient TBT concentrations of coastal waters. Ecotoxicology 9, 273-285.

Qun-Fang, Z., Gui-Bin, J., Ji-Yan, L., 2002. Effects of sublethal levels of tributyltin chloride in a new toxicity test organism: the Chinese rare minnow (Gobiocypris rarus). Archives of Environmental Contamination and Toxicology 42, 332-337.

Rosa, S.M.L.J., Antunes-Madeira, M.C., Jurado, A.S., Madeira, V.M.C., 2000. Amiodarone interactions with membrane lipids and growth of Bacillus stearothermophilus used as a model. Applied Biochemistry and Biotechnology 87, 165-175.

Rüdel, H., 2003. Case study: bioavailability of tin and tin compounds. Ecotoxicology and Environmental Safety 56, 180-189.

Schulte-Oehlmann, U., Oehlmann, J., Fioroni, P., Bauer, B., 1997. Imposex and reproductive failure in Hydrobia ulvae (Gastropoda: Prosobranchia). Marine Biology 128, 257-266.

Shinitzky, M., Barenholz, Y., 1978. Fluidity parameters of lipid regions determined by fluorescence polarization. Biochimica et Biophysica Acta 515, 367-394.

Sikkema, J., de Bont, J.A.M., Poolman, B., 1995. Mechanisms of membrane toxicity of hydrocarbons. Microbiological Reviews 59, 201-222.

Smith, L.D., Negri, A.P., Philipp, E., Webster, N.S., Heyward, A.J., 2003. The effects of antifoulant-paint-contaminated sediments on coral recruits and branchlets. Marine Biology 143, 651-657.

Stockdale, M., Dawson, A.P., Selwyn, M.J., 1970. Effects of trialkyltin and triphenyltin compounds on mitochondrial respiration. European Journal of Biochemistry 15, 342-351.

Tilley, L., Thulborn, K.R., Sawyer, W.H., 1979. An assessment of the fluidity gradient of the lipid bilayer as determined by a set of $n$ - $(9$ anthroloxy) fatty acids $(n=2,6,9,12,16)$. Journal of Biological Chemistry 254, 2592-2594.

Trotter, P.J., Storch, J., 1989. 3-[p-(6-phenyl)-1,3,5-hexatrienyl]phenylpropionic acid (PA-DPH): characterization as a fluorescent membrane probe and binding to fatty acid binding proteins. Biochimica et Biophysica Acta 982, 131-139.

White, J.S., Tobin, J.M., 2004. Inorganic tin and organotin interactions with Candida maltosa. Applied Microbiology and Biotechnology 63, 445-451.

White, J.S., Tobin, J.M., Cooney, J.J., 1999. Organotin compounds and their interactions with microorganisms. Canadian Journal of Microbiology 45, 541-554. 